\title{
Dietary supplementation of different parts of Andrographis paniculata affects the fatty acids, lipid oxidation, microbiota, and quality attributes of longissimus muscle in goats
}

\begin{abstract}
The effects of dietary supplementation of different parts of Andrographis paniculata on fatty acids, lipid oxidation, microbiota and quality attributes of Longissimus thoracis et lumborum (LTL) muscle in goats were assessed. Twenty four, entire Boer bucks (4 Thionths old;

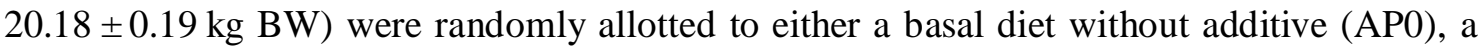

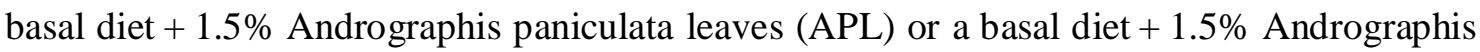
paniculata whole plant (APW). The bucks were fed the diets for $100 \mathrm{~d}$ and slaughtered. The LTL muscle was subjected to a $7 \mathrm{~d}$ chill storage. The AP0 meat had higher (p仰 concentration of C16:0 and C18:0 than the APW and APL meat. The concentrations of total C18:1 trans, total CLA, C18:1n-9, C18:2n-6, C18:3n-3 and C20:5n-3 were higher (p APL and APW meat than the AP0 meat. Diets had no effect (p\प05) on muscle glycogen, $\mathrm{pH}$, drip loss, chemical composition and lactic acid bacteria count. Cooking loss, shear force,

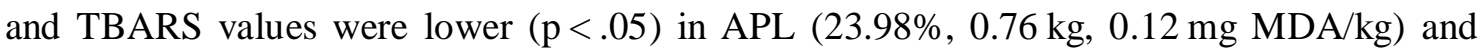
APW $(24.53 \%, 0.80 \mathrm{kgg}, 0.15$ The MDA $/ \mathrm{kg}$ ) meat compared with AP0 (26.49\%, $1.13 \mathrm{Wkg}$,

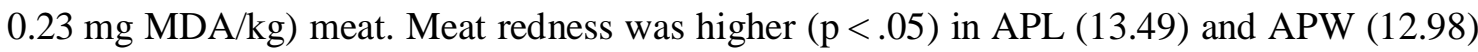
than AP0 (10.86). Sensory scores for juiciness, tenderness, and overall acceptability of APL $(7.92,7.88,7.89)$ and APW $(7.90,7.08,7.77)$ meat were higher $(\mathrm{p} \mathbb{R}[05)$ than that of the AP0 $(5.38,5.95,5.41)$ meat. Total viable counts and populations of Pseudomonas spp, Escherichia coli and Enterobacteriacea were higher $(\mathrm{p} \mathbb{R} \square(05)$ in AP0 meat than in APL and APW meat.

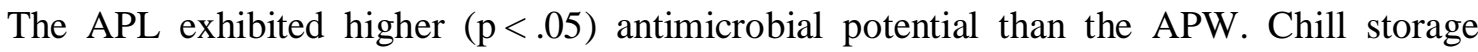
affected (p他D5) the physicochemical properties, lipid oxidation and microbial counts in chevon. Dietary APL and APW enhanced the beneficial fatty acids, quality attributes and oxidative stability, and reduced microbial counts in chevon.
\end{abstract}

Keyword: Cooking loss; Enterobacteriacea; Escherichia coli; Meat quality; Pseudomonas spp; Redness; Tenderness 